\title{
Design of Human Motion Capture System Based on Computer Vision
}

\author{
Wen-jing GUO ${ }^{1,2, a *}$, Yong-ren $\mathrm{CHEN}^{3, b}$, Shu-qing $\mathrm{CHEN}^{1, c}$, Xiu-li YANG ${ }^{1, d}$, \\ Lin-jing QIN ${ }^{1, e}$ and Jin-gang LIU ${ }^{1, f}$ \\ ${ }^{1}$ Department of Aircraft Dynamics, Air Force Aviation University of Chinese People's Liberation \\ Army (CPLA), 2222 Nanhu Road, Changchun, China, 130022 \\ ${ }^{2}$ Department of Engineering Mechanics, College of Mechanical Science and Engineering, Jilin \\ University, 5988 Renmin Street, Changchun, China, 130025 \\ ${ }^{3}$ Department of Aviation Lifesaving, Air Force Aviation University of the CPLA, 7855 Renmin Street, \\ Changchun, China, 130022 \\ axxguo@163.com, ${ }^{b}$ chenyr2011@163.com, ${ }^{c} 250098884 @ q q . c o m,{ }^{d} 11955504 @ q q . c o m$, \\ eqinlinjing2000@qq.com, ${ }^{\mathrm{T}} 27656670 @ q q . c o m$ \\ * Corresponding author
}

Keywords: Human motion capture, Computer vision, Camera calibration, 3D reconstruction. Abstract. Human motion capture is widely applied in engineering bionics, ergonomics, aerospace, military defense, biomedical, sports competition and other fields. However, the human motion capture equipments are less developed in China. This paper presents a human motion capture system based on computer vision using binocular camera. Stereo camera calibration is an important step in computer vision. It occupies the important status in stereo camera distance measurement, three-dimensional reconstruction and other fields. In this paper, a new method of binocular calibration is proposed on the basis of monocular camera. It can also get the translation and rotation between two cameras comparing with the traditional monocular camera calibration. Firstly, the binocular camera calibration method and the software platform framework of the system were presented. Eventually, the results of experiment were given to validate the correctness and effectiveness of the proposed method.

\section{Introduction}

The study of human movement is very important to some fields, such as engineering bionics, ergonomics, aerospace, military defense, biomedical, sports competition [1-7]. Image recognition method based on computer vision has been widely used in human motion tests $[8,9]$. The research goal of computer vision is to enable computers to perceive three-dimensional environment through the two-dimensional image, which can make some post-processing, such as three-dimensional reconstruction and visual position. Camera calibration accuracy directly affects the performance of vision measurement system. First, perform calibration individually for each camera, and then global calibration using various camera constraints, to improve accuracy of camera calibration [10]. Since computer vision belongs to reverse problem, the depth will be lost in projection, and subject to interference of light, material properties, human faces, and other physical factors. Current is lacking of understanding about visual and intelligence mechanism of human [11-13]. So, there is a considerable gap between computer vision and human vision. This paper presents a human motion capture system based on computer vision using binocular camera to solve the above problems.

\section{Materials and Methods}

Software platform framework of the system is as shown in Fig. 1.

1. The initial tracking point of video was confirmed in the initialization phase and the model parameters were initialized. 


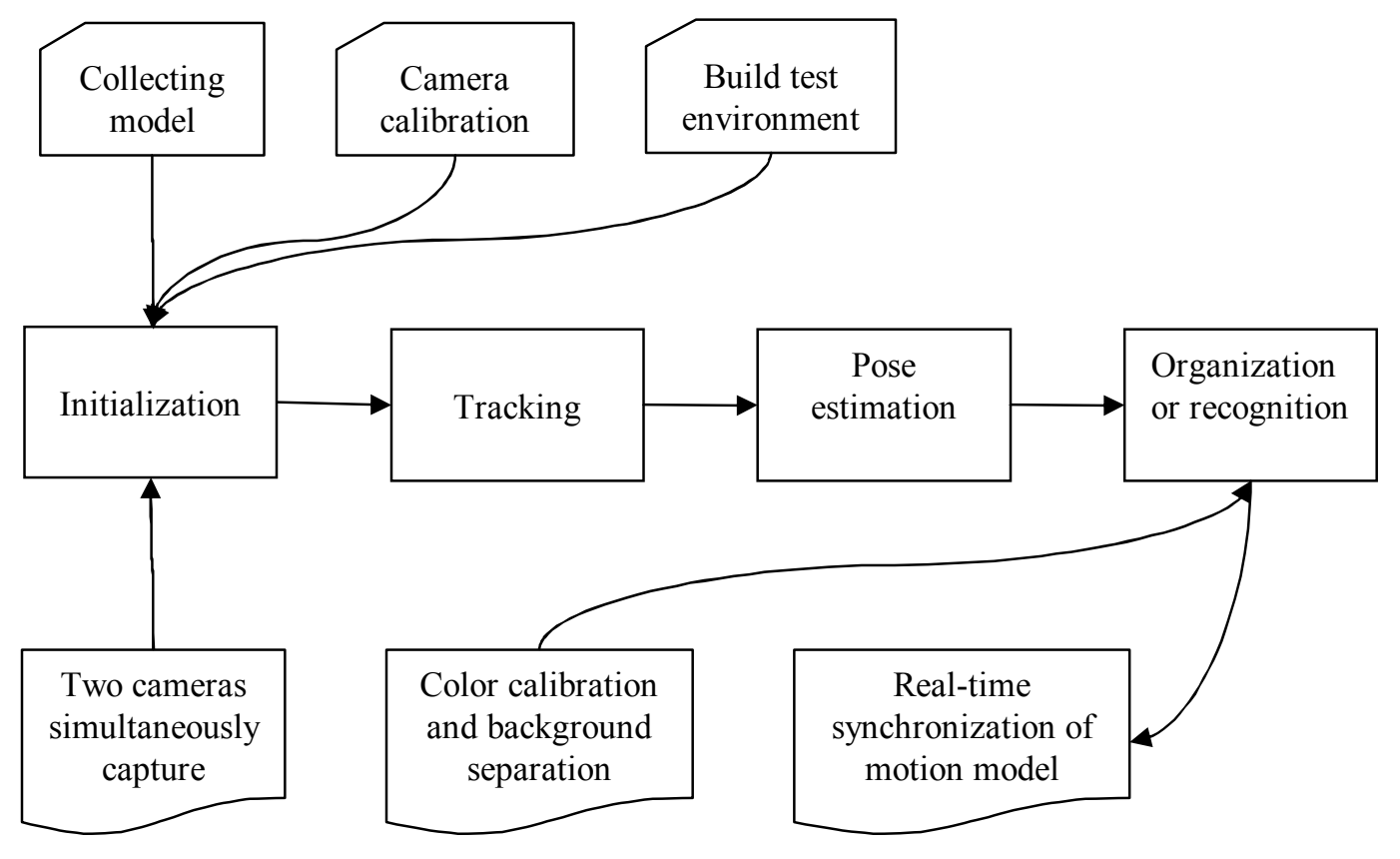

Fig. 1 Software platform framework of the system

(1) Collecting model

Skeleton and wireframe models were used in the system. The skeletal model was collected from the online open source model library of human skeleton. The wireframe model was generated in real time by video window.

(2) Camera calibration

The calibration method based on OpenCV was used. The inside and outside parameters of cameras were obtained by extraction of the corners of Othello checkerboard $8 \times 9(5 \mathrm{~cm} \times 5 \mathrm{~cm} /$ grid $)$.

(3) Build test environment

The binocular cameras were used avoiding direct natural light. The shooting background was as simple as possible. All three yellow and green papers $(5 \mathrm{~cm} \times 5 \mathrm{~cm} /$ sheet $)$ were respectively affixed to hip, knee and ankle joints of the body.

(4) Two cameras simultaneously capture

The network cables of two cameras simultaneously were connected the network port of frame grabber (Figure 2).

2. The feature objects were extracted on the basis of the timing attribute or spatial properties of video in the tracking phase.

3. The motion estimation was based on motion model in the pose estimation phase.

4. The de-noising, smoothing and formatting of motion data were completed in the organization or recognition phase. The legs actions were restored by serializing the data of motion capture.

(1) Color calibration and background separation

The position of landmarks was determined by the colors extraction through OpenCV function, clicking a color on the video image and ignoring background.

(2) Real-time synchronization of motion model

The window of skeleton model was popped-up by Setdlgitem function, the wireframe models were drawn by Showimage function, binding the coordinates of human space and models. 


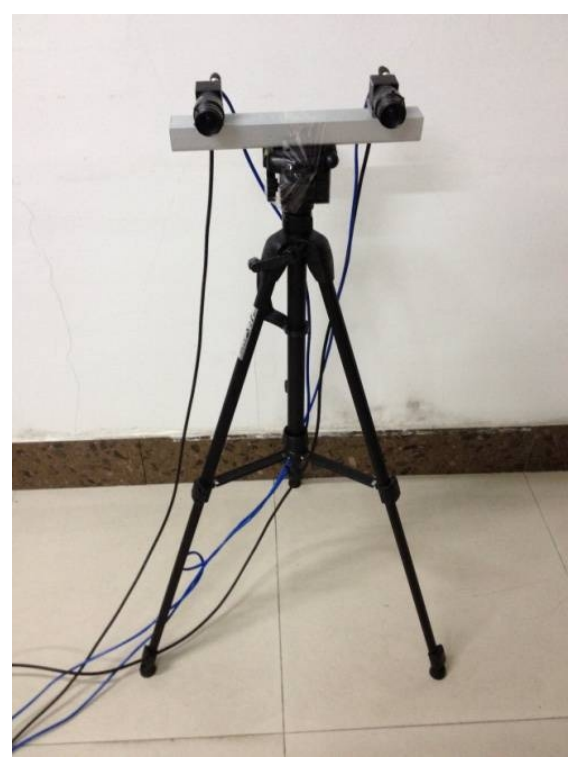

Fig. 2 Two cameras simultaneously capture

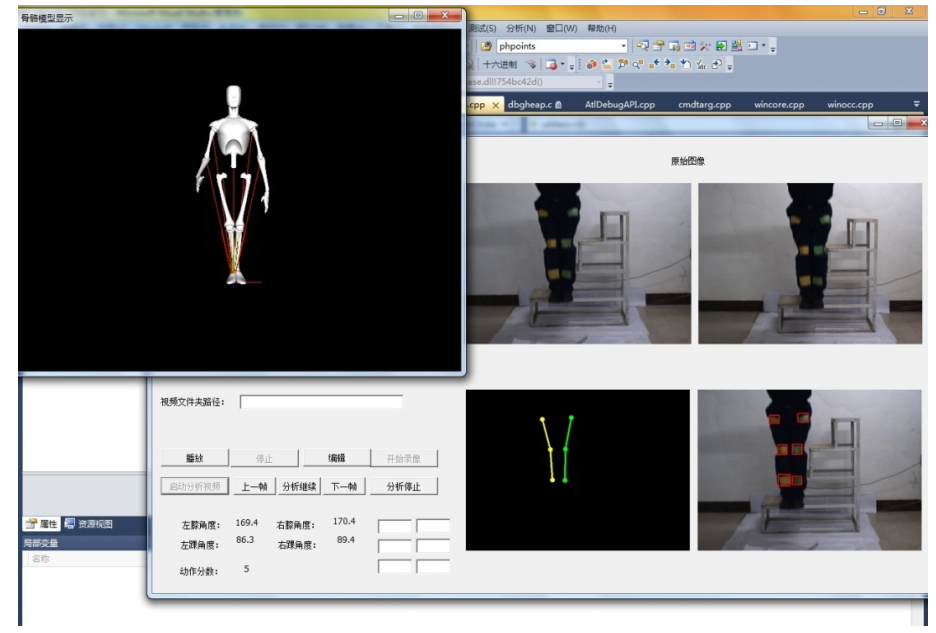

Fig. 3 Display function of the system

\section{Results and Discussion}

The system is able to display realistically three-dimensional human motion through wireframe model and skeletal model (Fig. 3). The body posture may manually edited by the system (Fig. 4). The knee flexion and ankle plantar flexion angles of human body may outputted by the system (Fig. 5).

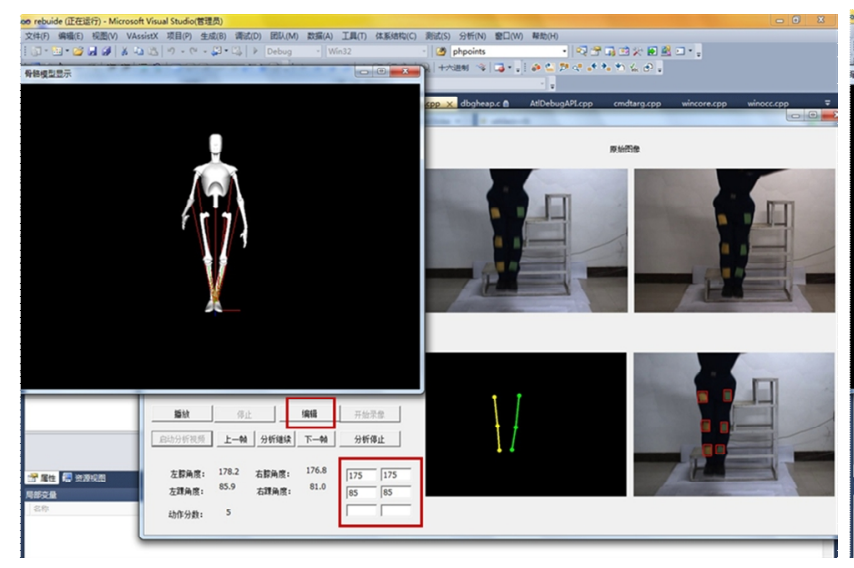

Fig. 4 Editing function of the system

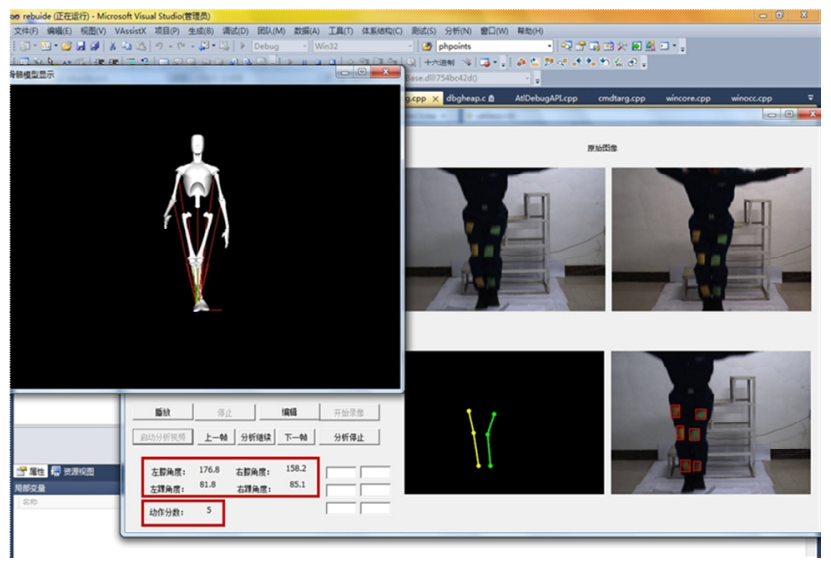

Fig. 5 Assessment function of the system

The system with parallel binocular camera does not capture the motion of side and back of human body. Increasing the number of cameras is proposed in order to capture more comprehensive of human movement from multiple perspectives. The resolution and shooting rate of the cameras in the system is low, which cause motion blur, and greater impact of messy background and illumination changes on the extraction of identification point. Infrared cameras may be used to improve the extraction accuracy of objects, which can expand the application scope of the system. We recommend that the system is connected force platform measuring ground reaction force in order to analyze the dynamics of human motion, further clarify the mechanism of biomechanics.

\section{Conclusions}

This human motion capture system very well complete its indicators, achieved better trial results, meet the basic requirements of human motion assessment, that can be further popularized in the relevant fields of biomechanics. At the same time the system confirmed the feasibility of constructing a 
three-dimensional human body model by the binocular based on extraction of color-coded identification point, which will promote the development of computer vision technology.

\section{Acknowledgements and Disclaimers}

This work was financially supported by the "five-second" scientific and technological research projects ("spring sprout talents scheme" special) named "Study on the biomechanical mechanism of knee injury during parachute landing" of Jilin Province Education Department of China. The opinions or assertions contained herein are the views of the authors and are not to be construed as official or as reflecting the views of the Air Force Department of the CPLA.

\section{References}

[1] Y. X. Lu: Journal of Bionics Engineering, Vol. 1 (2004), p.1

[2] W. B. Griffin, W. R. Provancher, M. R. Cutkosky: PRESENCE: Teleoperators and Virtual Environments, Vol. 14 (2005), p.720

[3] http://www.honda-taiwan.com.tw/tech/asimo/ASIMO01.html

[4] http://tech.163.com/tm/031219/031219_120775.html

[5] S. Huang: Human Movement Science, Vol. 17 (1998), p.821

[6] L. Ren, R. Jones and D. Howard: Journal of Biomechanics, Vol. 38 (2005), p. 853

[7] G. Zhao, L. Ren, L. Ren, J. R. Hutchinson, L. Tian, J. S. Dai: Journal of Bionic Engineering, Vol. 5 (2008), p. 328

[8] H. Ning, L. Wang, W. Hu, T. Tan: Proceedings of the 4th IEEE International Conference on Multimeia Interfaces, (2002), p.383

[9] http://www.motionanalysis.com.cn/application/film\%20and\%20art.htm

[10]Meng L, Bo Z, Zhengda M, Xianzhong D: Computer Technology and Development, Vol. 25 (2015), p.69

[11]Guoru Z: Doctoral Dissertation of Jilin University, (2009)

[12]Juxia L: Computer Simulation, Vol. 32 (2015), p.442

[13]Yupu Z, Qi Y, Qi Z: Computer Application, Vol. 35 (2015), p.502 